\title{
Unilateral balsalazide-induced eosinophilic pneumonia in an ulcerative colitis patient
}

\author{
${ }^{1} \mathrm{~J}$ McGrane, ${ }^{2} \mathrm{M}$ Paravasthu, ${ }^{3} \mathrm{~W}$ Candlish, ${ }^{4} \mathrm{~J}$ Gravil \\ ${ }^{1,2}$ ST2 in core medical training; ${ }^{3}$ Consultant Pathologist; ${ }^{4}$ Consultant in Respiratory Medicine, Respiratory Unit, Royal Alexandra Hospital, \\ Paisley, UK
}

\begin{abstract}
We report a case of unilateral eosinophilic pneumonia secondary to balsalazide monotherapy for ulcerative colitis. After commencing balsalazide, the patient presented with a history of cough, progressive dyspnoea and lethargy. Blood counts revealed peripheral eosinophilia. Her chest radiograph showed leftsided infiltration, and high-resolution computerised tomography demonstrated widespread nodular shadowing and ground glass opacifiction in the left lung. The right lung was normal. Transbronchial lung biopsy confirmed eosinophilic pneumonia. There was rapid clinical and radiological improvement after withdrawing balsalazide. Drug-induced eosinophilic pneumonia has been described with mesalazine and sulfasalazine, but no case reports of balsalazide-induced eosinophilic pneumonitis have been found.
\end{abstract}

KEYWORDS Balsalazide, drug-induced eosinophilic pneumonia, eosinophilic pneumonia, inflammatory bowel disease, ulcerative colitis

DECLARATION OF INTERESTS No conflict of interests declared.
Correspondence to J McGrane, Plymouth Oncology Centre, Derriford Hospital,

Plymouth PL6 8DH, UK

tel. +44 (0)7879 876639

e-mail

johnmcgrane2003@yahoo.co.uk

\section{CASE REPORT}

A 44-year-old woman with no medical history of note presented to her general practitioner with a one-month history of diarrhoea and rectal bleeding. Colonoscopy and biopsy diagnosed ulcerative colitis. She was commenced on balsalazide monotherapy with excellent clinical effect. She presented to the acute medical unit five weeks later with a four-week history of cough, increasing dyspnoea, lethargy, night sweats and weight loss. These symptoms started approximately one week after starting balsalazide therapy. There had been no improvement in symptoms after a week of oral cefalexin antibiotic therapy in the community.

Clinical examination at her presentation to hospital revealed pyrexia $\left(38.2^{\circ} \mathrm{C}\right.$ ), pulse rate 105 beats $/ \mathrm{min}$, oxygen saturation $96 \%$ on air and crepitations throughout the left hemithorax. Initial blood results showed a white blood cell count of $21 \times 10^{9}$ cells/l. Eosinophil counts were elevated, with counts of $5.25 \times 10^{9} / 1$ rising to $8.38 \times 10^{\circ} /$. Renal profile and liver function tests were unremarkable. A chest radiograph showed diffuse infiltration in the mid zone of the left lung (Figure I).

Intravenous fluid resuscitation and co-amoxiclav therapy were commenced on admission. Blood and sputum cultures grew no micro-organisms. Autoantibody and atypical pneumonia (including tuberculosis) screening were negative.

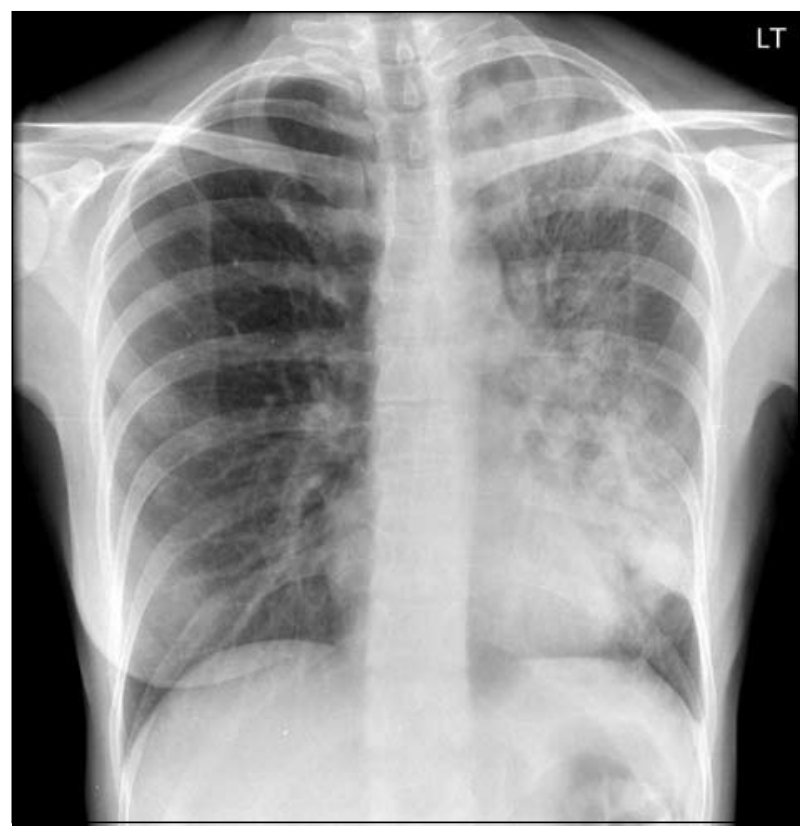

FIGURE I Chest radiograph showing patchy infiltrates in the left lung.

The patient had no symptoms of diarrhoea or rectal bleeding to suggest an exacerbation of her ulcerative colitis, and an abdominal examination was normal.

After 48 hours, eosinophilic pneumonia (EP) was suspected due to the limited response to antibiotics, increasing peripheral eosinophilia and the correlation of symptoms with the commencement of new medication. 
A high-resolution computed tomography (CT) scan confirmed widespread, predominantly centrilobular, nodular shadowing and ground glass opacifiction in the left lung. No abnormalities were demonstrated in the right lung (Figure 2).

A transbronchial lung biopsy showed eosinophilic pneumonia with no evidence of arteritis or granuloma formation. Immunohistochemistry did not demonstrate the presence of Langerhans cells within the alveolar infiltrate (Figure 3).

Twenty-four hours after discontinuing balsalazide there was clinical improvement, leading to a complete resolution of symptoms within two weeks. Four weeks post discharge, the patient's eosinophilia had resolved and serial chest radiographs showed radiological resolution (Figure 4). She did not require corticosteroid therapy during her admission.

Since the cessation of balsalazide the patient has not had any recurrence of these respiratory symptoms to date.

\section{DISCUSSION}

Sulphasalazine, sulphapyridine bound to 5-aminosalicylic acid (5-ASA), and 5-ASA drugs such as mesalazine are established therapeutic agents in the treatment of inflammatory bowel diseases (IBD). Balsalazide is a prodrug of mesalazine consisting of a carrier molecule linked to mesalazine by an azo bond. It is converted to mesalazine by the bacterial action in the colonic lumen and has a local lumenal anti-inflammatory action. It is reported to have a better tolerability profile than sulphasalazine and is an effective first-line therapeutic agent in the treatment of ulcerative colitis (UC).' Symptomatic improvement is seen earlier than with similar treatment with mesalazine, and high-dose balsalazide has been reported as being superior at preventing relapses compared with mesalazine in the treatment of UC. ${ }^{2,3}$

Both sulphasalazine and mesalazine have been reported as causing pulmonary toxicity during treatment of IBD. A review of the literature shows 38 cases of pulmonary toxicity related to the 5-ASA drug group, including EP, eosinophilic pleural effusion, organising pneumonia, bronchospasm, chest pain and interstitial lung disease with a granulomatous pattern. ${ }^{4}$ Approximately ten cases of EP associated with mesalazine in the treatment of inflammatory bowel disease have been reported in English-language publications. One report of unilateral eosinophilic pneumonia related to mesalazine was identified. This case had a similar presentation to our patient and resolved following the withdrawal of mesalazine and a tapering dose of steroids. ${ }^{5}$.

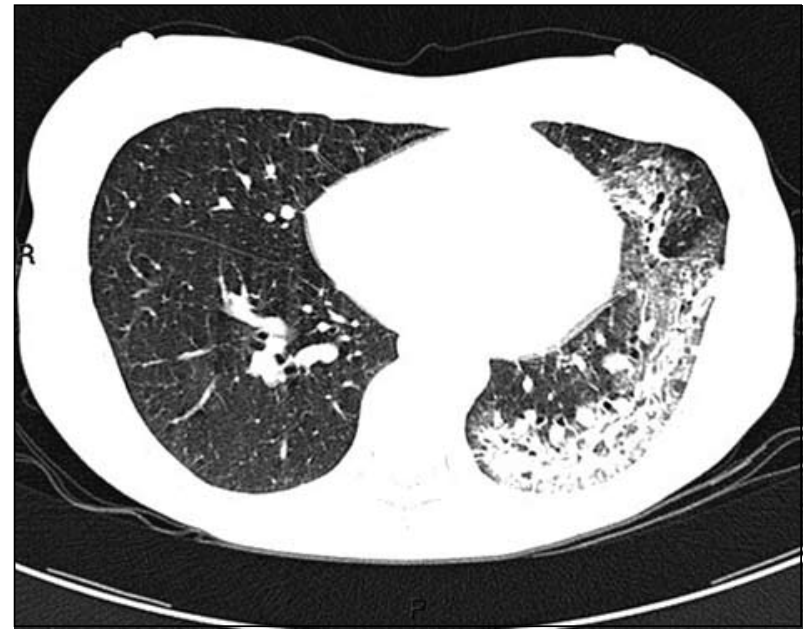

FIGURE 2 High-resolution CT scan showing nodular shadowing with ground glass opacification and volume loss in the left lung.

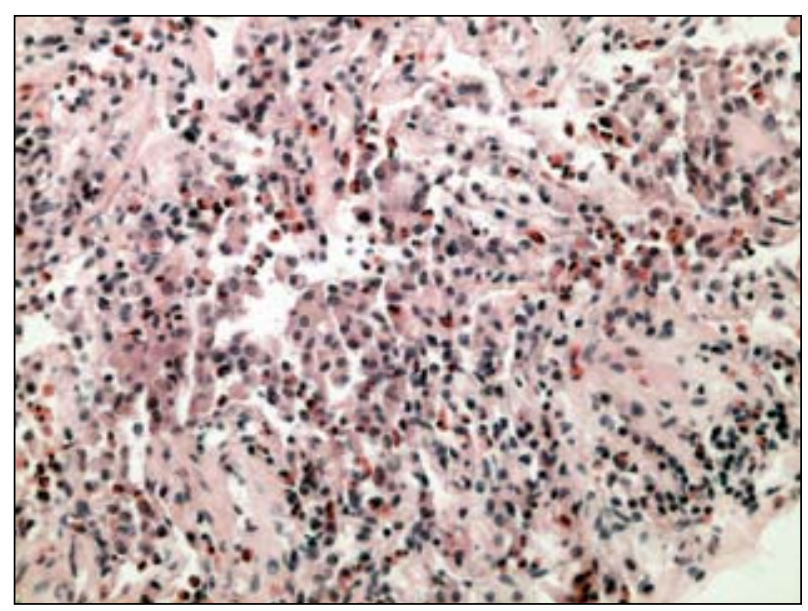

FIGURE 3 Slide from the patient's transbronchial biopsy showing eosinophilic infiltration (haematoxylin and eosin stain, magnification $\times 100$ ). Eosinophils are seen as red-staining cells.

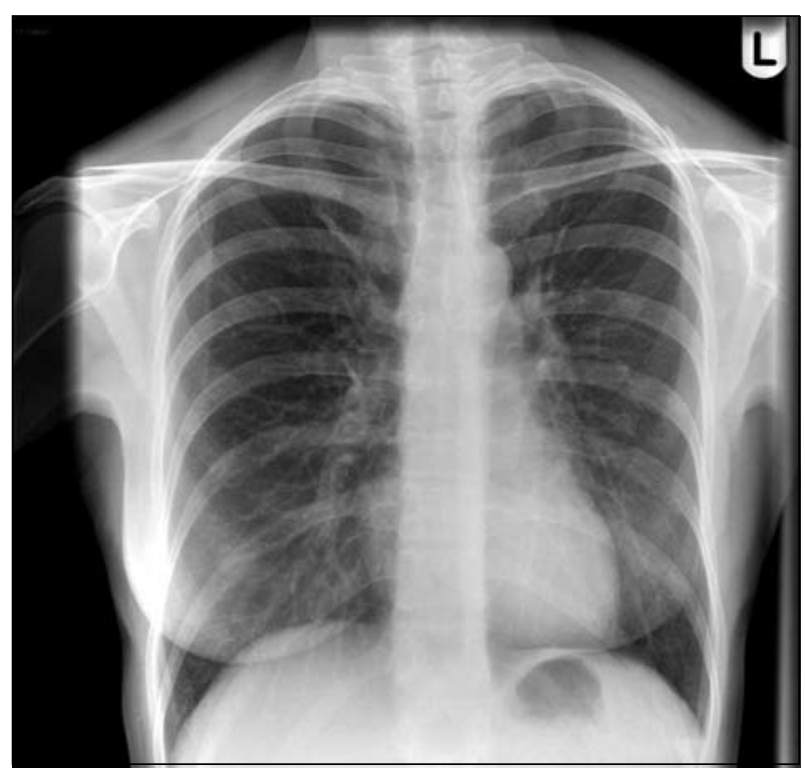

FIGURE 4 Chest radiograph six weeks after withdrawing balsalazide treatment. 
Given that mesalazine is the active agent in balsalazide we would have expected to find reports of pulmonary toxicity associated with balsalazide. On reviewing the literature we found no such reports.

5-aminosalicylic acid drug-induced lung toxicity and other drug-induced EPs manifest as new onset dyspnoea, cough and fever associated with pulmonary infiltrates. A review of 14 cases of high-resolution $C T$ findings in drug-induced EPs showed the majority had centrilobular nodules and all had bilateral pulmonary infiltrates. These infiltrates were usually ground glass opacities and consolidation involving the outer third of the lungs. ${ }^{8}$ High-resolution CT clearly reveals the pattern of lung involvement, which may include areas of ground glass opacities, nodules, consolidation and irregular lines.

Diagnosing drug-induced EPs can be difficult and often requires confirmation by means of bronchoalveolar lavage and transbronchial biopsy. Symptoms should resolve within several weeks of drug withdrawal, which remains the mainstay of management.

Although corticosteroids are often used, their efficacy remains uncertain in drug-induced EP. If a cessation of the drug does not lead to improvement, a trial of corticosteroids could be considered.

Our case presented with many of the clinical features of drug-induced EP described above. Transbronchial biopsy was performed due to the unilateral presentation and to differentiate between drug-induced EP and pulmonary manifestations of UC. The clinical course and the fact that the patient has had no return of her respiratory symptoms are both consistent with drug-induced EP.

\section{CONCLUSION}

As of November 2009, approximately 119 drug groups have been reported as causing eosinophilic lung disorders. This wide range includes antibiotics, antihypertensives, antidepressants and cytotoxic medications. ${ }^{4} \mathrm{~A}$ high index of clinical suspicion should be maintained, especially in the presence of respiratory symptoms and peripheral eosinophilia after starting a new medication.

We report this case to make clinicians aware of potential lung toxicity with balsalazide, and that druginduced eosinophilic pneumonias may present with unilateral involvement.

\section{REFERENCES}

I Muijsers RB, Goa KL. Balsalazide: a review of its therapeutic use in mild-to-moderate ulcerative colitis. Drugs 2002; 62:1689-705. doi:I0.2165/00003495-200262II0-00010

2 Pruitt R, Hanson J, Safdi $M$ et al. Balsalazide is superior to mesalamine in the time to improvement of signs and symptoms of acute mild to moderate ulcerative colitis. Am J Gastroenterol 2002; 97:3078-86. doi:I0. I I II/j. I572-024I.2002.07I03.x

3 Kruis W, Schreiber S, Theuer D et al. Low dose balsalazide ( $1.5 \mathrm{~g}$ twice daily) and mesalazine ( $0.5 \mathrm{~g}$ three times daily) maintained remission of ulcerative colitis but high dose balsalazide ( $3.0 \mathrm{~g}$ twice daily) was superior in preventing relapses. Gut 2001; 49:783-9. doi:I0.II36/gut.49.6.783

4 Foucher P, Camus P. Pneumotox on line: the drug-induced lung diseases. Available from: http://www.pneumotox.com

5 Saltzman K, Rossoff LJ, Gouda $\mathrm{H}$ et al. Mesalamine-induced unilateral eosinophilic pneumonia. AJR Am J Roentgenol 200I; I77:257.

6 Parry SD, Barbatzas C, Peel ET et al. Sulphasalazine and lung toxicity. Eur Respir J 2002; 19:756-64. doi:10.1 I83/09031936.02.00 267402

7 Tanigawa K, Sugiyama K, Matsuyama H et al. Mesalazineinduced eosinophilic pneumonia. Respiration 1999; 66:69-72. doi:I0.II59/00002934I

8 Souza CA, Müller NL, Johkoh T et al. Drug-induced eosinophilic pneumonia: high-resolution CT findings in 14 patients. AJR Am J Roentgenol 2006; 186:368-73. doi:I0.22 I4/AJR.04.1847

9 Jeong YJ, Kim KI, Seo IJ et al. Eosinophilic lung diseases: a clinical, radiologic, and pathologic overview. Radiographics 2007; 27:61739. doi: I0.I |48/rg.27306505 I

\section{CONFERENCING AND EVENTS}

The Royal College of Physicians of Edinburgh has a unique blend of rooms providing the perfect location for your conference, meeting or event. The Victorian Great Hall, galleried New Library and the Georgian Cullen Suite are wonderful settings for dinners and receptions. The modern Conference Centre seats up to 300 people in raked seating and is complemented by breakout rooms seating from 10 to 150 people, a keypad voting system and video conferencing. The College provides a stunning setting for weddings and receptions and is licensed for both civil and religious ceremonies. Discounts are available for Fellows and Members, medical conferences and charities.

For more information or for a quotation, please contact the Events Team on +44 (0)।3। 225 7324; email events@rcpe.ac.uk or visit http://www.rcpe.ac.uk/conferencing/index.php

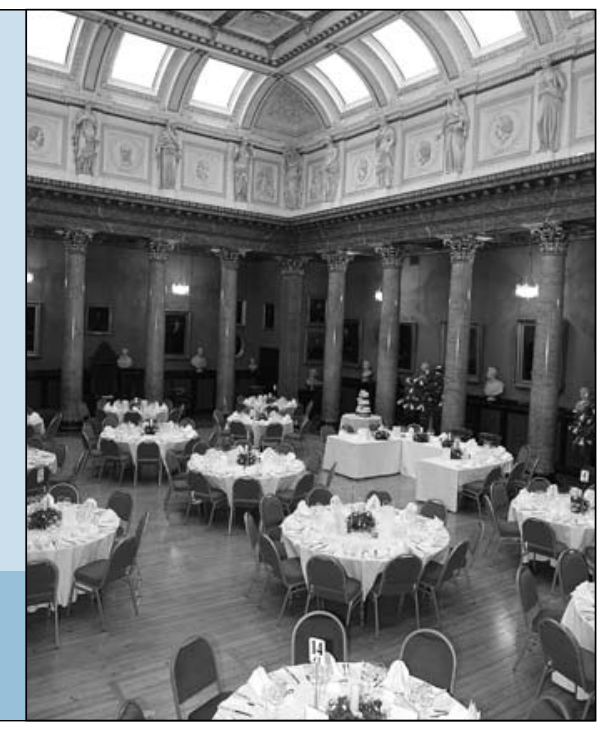

